\title{
Image Contrast Enhancement Based on Block-Wise Intensity-Pair Distribution with Two Expansion Forces
}

\author{
Md. Hasanul Kabir, M. Abdullah-Al-Wadud, and Oksam Chae* \\ Department of Computer Engineering, Kyung Hee University, \\ 1 Seochun-ri, Kiheung-eup, Yongin-si, \\ Kyunggi-do, Korea, 446-701 \\ hasanul@khu.ac.kr, awsujon@yahoo.com, oschae@khu.ac.kr
}

\begin{abstract}
In this paper, we present a block overlapped intensity-pair distribution based image enhancement algorithm. Instead of using the intensitypair distribution of the whole image, this proposed algorithm takes the intensity-pair distribution block-wise and maps the intensity of the center pixel according to an expansion function. Analyzing the intensity difference of the intensity-pair, two different expansion force sets are generated for contrast stretch: one for soft edges, another for strong edges. In addition, a set of antiexpansion force is generated for smooth regions to avoid noticeable change. The contrast stretch and over-enhancement are controlled with a linear magnitude mapping function instead of a non-linear one. This linear mapping preserves the relative contrast enhancement ratio between the gray levels. The local information from blocks easily facilitates the contrast enhancement, brings out subtle edge information, and removes noises from the image.
\end{abstract}

Keywords: Anti-expansion force, expansion force, intensity pair.

\section{Introduction}

Contrast enhancement is an important issue in image processing for both human and computer vision. It is widely used for medical image processing and as a preprocessing step in speech recognition, texture synthesis, and many other image/video processing applications [4-7]. A very popular technique for contrast enhancement of images is histogram equalization (HE) [9-11]. It is the most commonly used method due to its simplicity. HE performs its operation by remapping the gray levels of the image based on the probability distribution of the input gray levels [8]. The basic idea behind this method is to stretch the dynamic range of the image histogram and resulting in overall contrast improvement. Many researches have already been done on histogram equalization and many methods have already been proposed. Generally, we can classify these methods in two principle categories global and local histogram equalization [12].

Global Histogram Equalization (GHE) uses the histogram information of the entire input image for its transformation function. Fig. 1(b) shows the result of GHE on the

\footnotetext{
Corresponding author.
} 
original image as shown in 1(a). Though this global approach is suitable for overall enhancement, it fails to adapt with the local brightness features of the input image [12] and shifts the brightness to the middle gray level of the image regardless of the input brightness [10]. Local histogram equalization (LHE), also known as Adaptive histogram equalization (AHE) [11], can get rid of such problem. It uses a small window that slides through every pixel of the image sequentially and only the block of pixels that fall in this window are taken into account for HE and then gray level mapping for enhancement is done only for the center pixel of that window. Thus, it takes the advantages of $\mathrm{HE}$, also remarkable use of local statistical information of the input image. But LHE requires high computational cost and sometimes causes overenhancement in some portion of the image. Another problem of this method is that it also enhances the noises in the input image along with the image features. To get rid of the high computational cost, another approach is to apply non-overlapping block based HE. Nonetheless, most of the time, these methods produce an undesirable checkerboard effects on enhanced images [9].

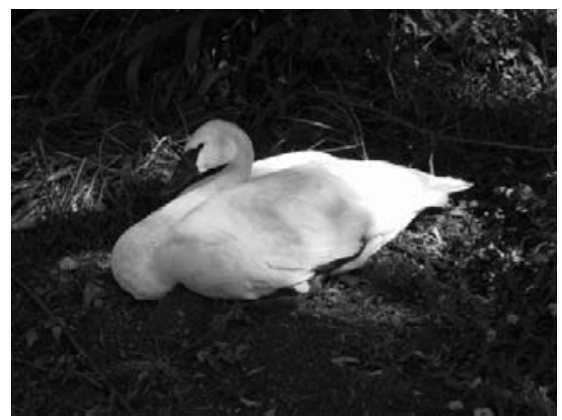

(a)

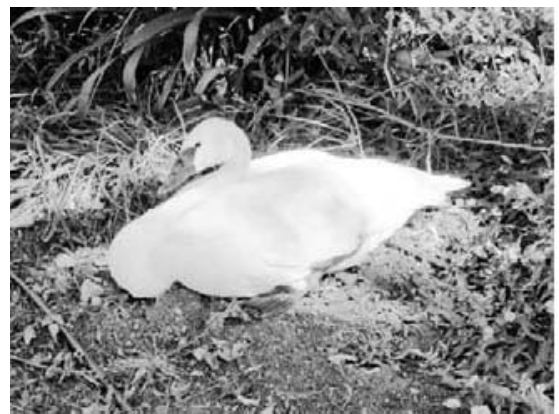

(b)

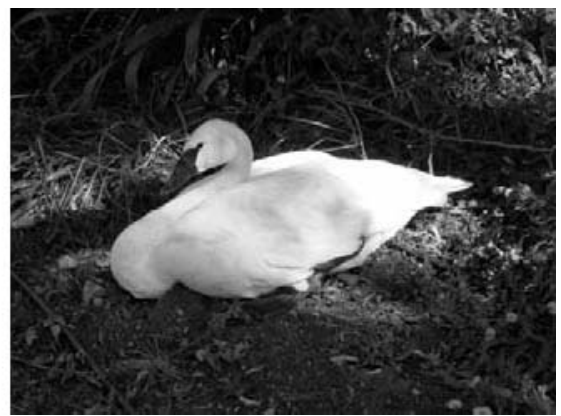

(c)

Fig. 1. (a) Original image. (b) Enhanced image resulting from GHE. (c) Enhanced image resulting from intensity-pair distribution based method.

Recently, [2] proposes curvelet based method for contrast enhancement. Though in multi-scale edge enhancement, curvelet based approach outperforms wavelet approach [3] in edge representation, but for noiseless or near noiseless images its enhancement is not remarkly better than wavelet based enhancement [2]. On the other 
hand, [1] proposes an approach for enhancement based on intensity-pair distribution that possesses both the local and global information of the image content. Depending on the intensity difference in the intensity pair, either a set of expansion forces or a set of anti-expansion forces is generated to get an intensity mapping function, which enhances contrast and suppresses the image noise in the output image. This mapping function is more or less like GHE mapping function but incorporating some neighborhood information from intensity pairs. However, still this method cannot sharpen edges with low intensity-pair contribution and remove impulse noises.

In this paper, our proposed algorithm exploits the block-wise intensity-pair distribution for edge enhancement. Generally, digital images contain two-dimensional array of intensity values with locally varying statistics, which results from different combinations of abrupt features, like edges and contrasting homogeneous regions [9]. Since different parts of the image have different statistical characteristics we apply the block based approach to handle local information effectively and leave less chance for the smooth regions from other part of the image to have more influence over the edge pairs and finally lead to better image contrast stretch. To control the contrast stretch of the soft edges and strong edges two different expansion forces are used, where the expansion force for the soft edge pairs are comparatively less than that of strong edge pairs. Unlike [1], where a nonlinear magnitude function was used to avoid overenhancement and preserve natural look of the processed image, here we present a linear magnitude mapping function to keep the relative contrast enhancement ratio between the gray levels since our search space is small and limited inside the block only. Once we get the final intensity mapping function from the expansion and anti-expansion forces of intensity pairs, only the center pixel of that block is updated and we move to the next pixel to repeat the same procedure. To take care of noises, if the deviation of center pixel's intensity value from its block intensity mean is too high, we replace that intensity value with the mean intensity value of that block, hence suppressing the noise pixels. Here we combined the advantages of conventional intensity-pair distribution method and block based approach for better edge extraction and enhanced image.

This paper is organized as follows. In section 2, we briefly introduce the intensitypair distribution based image enhancement method [1]. The proposed algorithm is explained in details in section 3. Section 4 lists a few experimental results on different images to illustrate the performance of the proposed approach while section 5 concludes this paper.

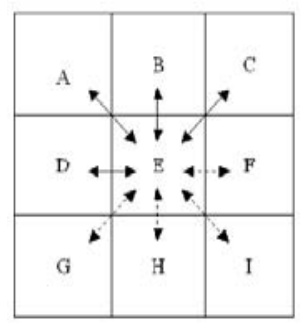

Fig. 2. An illustration of a pixel and its 8-neighbors. The solid arrows show the 4-neighbors from pixel $\mathrm{E}$ for intensity-pair generation. 


\section{Intensity-Pair Distribution Based Method}

This section will briefly describe the Intensity-Pair distribution method for image enhancement. The algorithm presented in [1] extracts both the global and local information. It starts with computing the intensity-pair distribution. For a given image, each pixel is checked with its 8-connected neighbors. Due to the commutative property of intensity pair, only 4-neighboring pixels in raster order are scanned. Fig. 2 shows an illustration of 4-neighbors taken into account for the pixel at $\mathrm{E}$ from its 8connection neighbor. Now to find intensity pairs belonging either to the smooth region or the edge region, we take the intensity difference within the pair. If the intensity difference is above a predefined threshold we can treat that pair as an edge pair otherwise that belongs to a flat region. To increase the contrast of the image and make the edges sharp we want to stretch the intensity of the edges pairs. On the contrary we also want to keep the flat regions from being stretched.

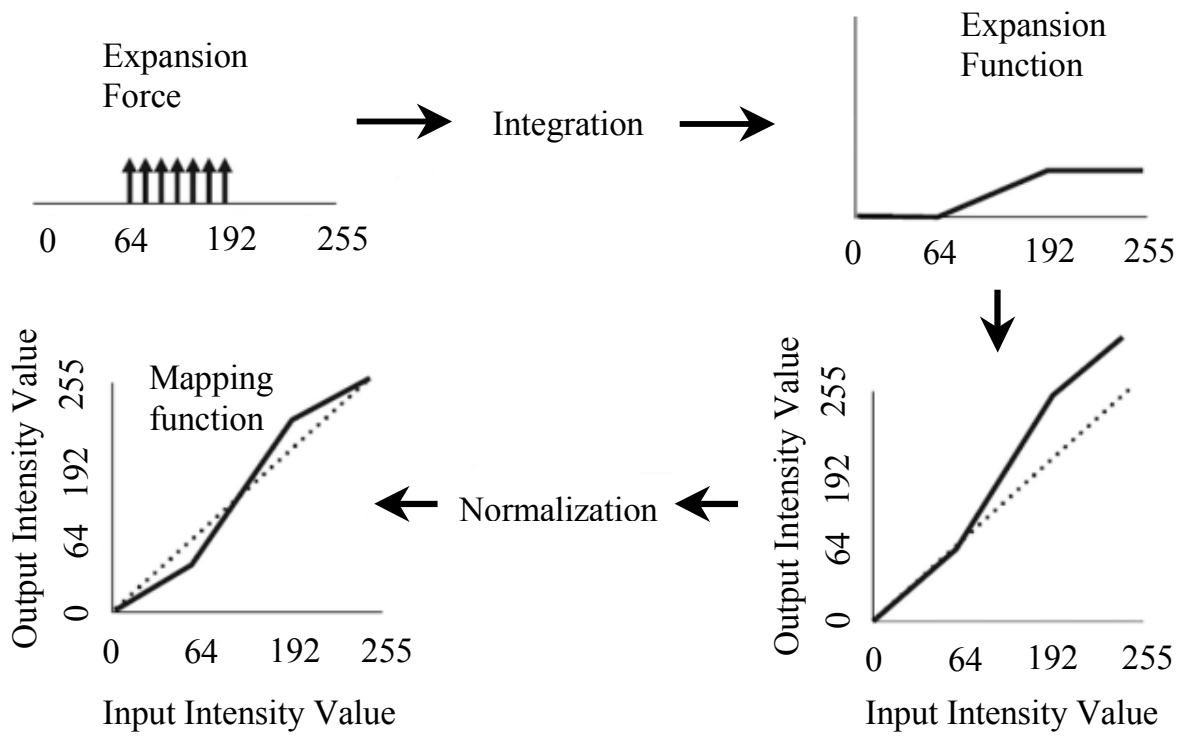

Fig. 3. Intensity mapping function generation procedure

In order to have contrast stretch in the edge pairs, we give a train of expansion forces between the gray level range of the edge pair. In a real 2D image many edge exists. So, we accumulate all the corresponding expansion forces of the edge pairs. Similarly all the anti-expansion forces are generated and accumulated for the intensity pairs of the smooth region. Now the smooth intensity pairs might lie within the gray level range of the edge pair. Due to the contrast stretch of the edge pair, the dynamic range of those smooth regions will also be stretched. To avoid these circumstances, the anti-expansion forces are subtracted from the expansion forces; hence the netexpansion force is obtained. Here, the anti-expansion forces preserve the smoothness for flat regions in the net-expansion force. Still the magnitude of the net-expansion 
force might be high enough to cause some unnatural and overly enhanced image. To reduce the dynamic range of the net expansion force a magnitude mapping function $\mathrm{M}($.$) is applied which constrains the expansion force to an extent so that the image$ looks natural. A proposed mapping function is

$$
Y=M(X)=X^{1 / m} .
$$

where, $X$ is the net-expansion force, $m$ is the behavior controlling parameter of the mapping function, and $Y$ denoting adjusted net-expansion force. Finally the expansion forces are integrated and normalized to get the expansion function for intensity mapping. Fig. 3 shows the procedure for generation of intensity mapping function and Fig. 1(c) shows the enhanced image resulting from using the just reviewed approach. Notice that the contrast of some of the edges have improved while some others have not improved significantly. This happened because the contrast stretch was done based on the global information irrespective of contrast stretch needed only for a particular region.

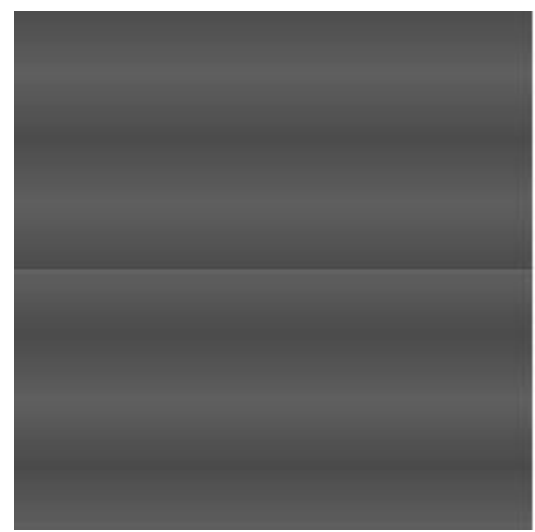

(a)

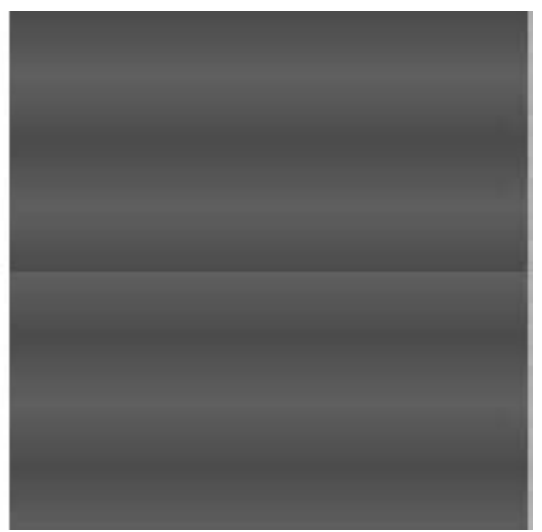

(b)

Fig. 4. (a) Original image. (b) Image resulting from intensity-pair distribution based method, which shows no enhancements made.

\section{Proposed Method}

In the proposed method, our key objective is to bring out the subtle details of the image by letting the less frequent intensity pairs contribute almost equally to the expansion function. In case of global intensity-pair distribution based method, there are chances that the gray levels of intensity pairs of smooth region might lie and cover the entire gray level range which the edge pairs are expecting to stretch. Since the smooth intensity pairs will generate trains of anti-expansion force, and due to the high occurrences of those pairs the anti-expansion force might nullify the expansion force. As a result, in the net-expansion force there will be no effect from those edge pairs and eventually no contrast stretch. Such an example is shown in Fig. 4. In the original image we have only $(0,48)$ edge pairs because there is sudden intensity jump from 0 
to 48 at the middle of the image. Rest of the image contains gray values gradually either increasing from 0 to 48 or decreasing from 48 to 0 . So our smooth intensitypairs are $(0,0),(0,1),(1,1),(1,2), \ldots,(47,48),(48,48)$. Thus the gray level ranges of the smooth regions have covered the entire gray level range of the edge pairs. Now though the edge pairs are generating expansion forces between its gray level ranges, due to high occurrences of smooth pairs the anti-expansion force generated by them will cancel the expansion force. In the end, no enhancement is done. But in case of block-based intensity pair distribution, since we focus only to the intensity pairs falling inside the block, there is no scope for the smooth regions to dominate the edge pair forces. When the block slides over the edges pixels, we have more edge pairs, which then give more expansion force at edge regions. This brings out fine edge information without having any threat from smooth regions. Again, when dealing with edges, we get two types of edges: soft edges and strong edges. Now if the same set of expansion force is applied for both soft edges and strong edges, we might get contrast stretch in intensity but not visually pleasing image. So we give a comparatively low expansion force to the soft edge pairs compared to the strong edge pairs. Low expansion force prevents the soft edges from being too sharp.

Moreover, to avoid unnatural or overly enhanced features due to the large magnitude expansion force, the contrast enhancement approach based on global intensity-pair distribution [1] uses a magnitude mapping function on the net expansion force, which is nonlinear in nature. Due to the nonlinearity, after applying the magnitude mapping function the ratios between the forces at different gray levels do not remain the same as before. So the resultant net expansion forces do not reflect properly the forces of the intensity pairs anymore. Since our proposed method confines attention only inside the block and we have less number of intensity pairs, intuitively there is less scope of having net-expansion force with extremely large magnitude. Here the proposed contrast enhancement algorithm makes use of a linear magnitude mapping function to compress the dynamic range of the net expansion forces and to keep the relative contrast enhancement ratio between the gray levels which otherwise will be lost incase of non-linear mapping function.

In our algorithm, the first step is to define a block and retrieve its intensity pair distribution. This distribution gives us the local information. If an edge falls inside the block then we will have many edge pairs. The expansion force and anti-expansion forces are computed in the same manner as discussed in the previous section with an additional check for soft edge and strong edge. To compress the dynamic range of the net expansion forces, we use a linear magnitude mapping function. An example of such a mapping function $\mathrm{M}($.$) is$

$$
Y=M(X)=X / \max \left(f_{0}, f_{1}, \ldots, f_{i}, \ldots, f_{255}\right) .
$$

where $f_{i}$ is the number of times expansion force is added for the $i$-th gray level. The next step is to integrate the net-expansion force to generate the expansion function and normalize it to fit in the range 0 to 255 . This function is used as the final intensity mapping function for the center pixel of the block. The presented approach also takes care of impulse noise pixels by computing the mean $(\mu)$ and standard deviation $(\sigma)$ of 
the block intensity values. For noise pixel removal, if the deviation of the center pixel's intensity value from its block mean is larger than twice the standard

1) Define an $M \times N$ sized output image array for an $M \times N$ input image $I$.

2) FOR each pixel position $(x, y)$ in the input image

a. Scan through every pixel within the block centered at pixel position $(x, y)$ to calculate the intensity-pair information, mean $\mu$, and standard deviation $\sigma$.

b. IF $|\mathrm{I}(\mathrm{x}, \mathrm{y})-\mu|>2 * \sigma$

THEN

Put $\mu$ as the intensity value at pixel position $(x, y)$ of output image and move to step 2 .

c. FOR each intensity-pair

IF Intensity difference $>$ Smooth region Threshold AND Intensity difference $>$ Soft edge Threshold

THEN

Train of expansion forces for strong edges is generated between the gray level range of intensity-pair.

ELSE IF Intensity difference $>$ Smooth region Threshold AND Intensity difference $<=$ Soft edge Threshold

THEN

Train of expansion forces for soft edges is generated between the

ELSE gray level range of intensity-pair.

THEN

Train of anti-expansion forces is generated between the gray level range of intensity-pair.

d. Accumulate the expansion and anti-expansion force.

e. For each gray level k, calculate the net expansion force based on the following equation:

\section{NetExpansionForce $[k]=$ ExpansionForce $[k]-$ $g \times$ AntiExpansionForce $[k]$}

here, $\mathrm{g}$ is chosen to be 0.1 empirically. If the net expansion force at $k$ is negative, reset that value to zero.

f. Apply the magnitude mapping function $\mathrm{M}$ (.) over the net expansion force.

g. Integrate the net expansion force to obtain the expansion function $\mathrm{T}$ and normalize it to fit within the range 0 to 255 .

$\mathrm{h}$. The intensity value for the output image at pixel position $(\mathrm{x}, \mathrm{y})$ is calculated using the following equation:

$$
w \times T[I(x, y)]+(1-w) \times I(x, y)
$$

here, $w$ is a combination factor with $0 \leq w \leq 1$

Fig. 5. Summary of the proposed algorithm

deviation of the block intensity value, the mean value is used instead of using the intensity mapping function-mapped value. Since only the pixels inside the block are 
taken into account for the generation of the intensity mapping function and computation of center pixel's intensity, we are dealing more with the local information. Next, the block is moved by one pixel and the same procedure is repeated until the end of the image is reached. Here contrast enhancement based on intensity-pair distribution is performed with each block information so it can adapt well to the subtle edge enhancement and partial light condition in the same way the block overlapped histogram equalization works. The proposed algorithm is basically a block overlapped intensity-pair distribution based image enhancement approach with two expansion forces and a linear magnitude mapping function. This idea is summarized more clearly in Fig. 5. In the next section, we will present a detailed experimental result to further display the effectiveness of the proposed algorithm.

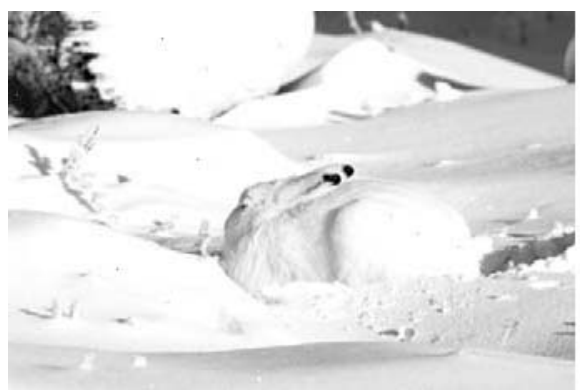

(a)

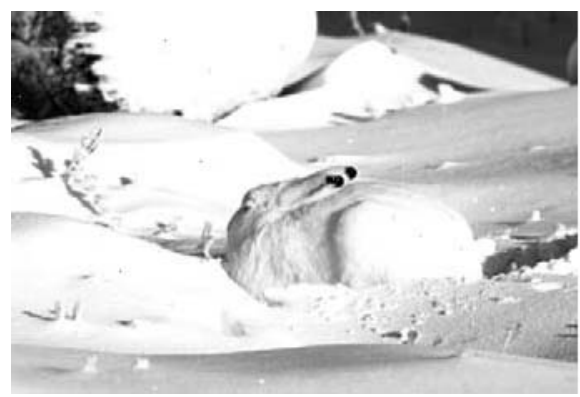

(c)

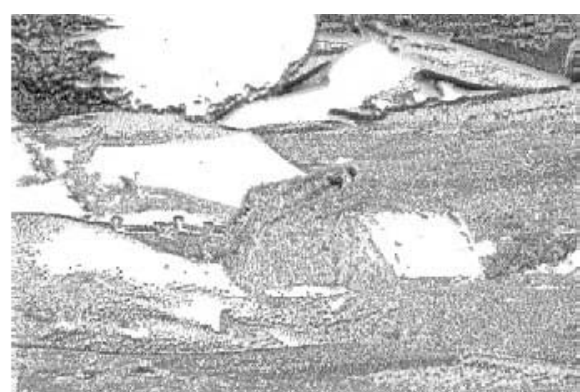

(b)

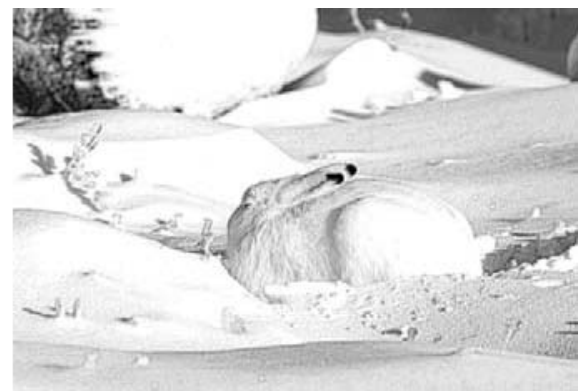

(d)

Fig. 6. (a) Original image (b) Enhanced image by LHE (c) Enhanced image by intensity-pair distribution based method with $(\mathrm{m}, \mathrm{g}, \mathrm{k})=(2,0.1,0.8)$ (d) Enhanced image by block based intensity- pair distribution with $(\mathrm{g}, \mathrm{w})=(0.1,0.6)$ and block size $3 \times 3$

\section{Experimental Results}

The results from previous algorithms and the proposed algorithm are simulated on various images, and compared with the enhancement ability of the proposed approach. Fig. 6 shows the original image along with simulation results from LHE, Intensity-Pair Distribution method and the proposed method. Here Block based Intensity-Pair distribution method has given better enhancement of the image, 
especially with the hare. Moreover, in the original image there are some impulse noises, which still remain for LHE and Intensity-Pair distribution method. Whereas Fig. 6(d) clearly shows our approach has quite efficiently removed those impulse noises.

To further prove the performance of the proposed algorithm, simulation result from applying the proposed method $(\mathrm{g}=0.1, \mathrm{w}=0.6$ and block size $3 \times 3)$ on satellite image is shown in Fig. 7(c), compared with Fig. 7(b) resulting from curvelet transform (c = $3,1=p=0.5$ and $s=0$ ). Here the edges and contrast at the upper portion are much more distinct in case of the enhanced image resulting from the proposed algorithm. Moreover, curvelet transform has also increased the overall brightness of the images, which might not be desirable in other images. In edge representation, image brightness and contrast, our proposed approach has done a significant improvement.

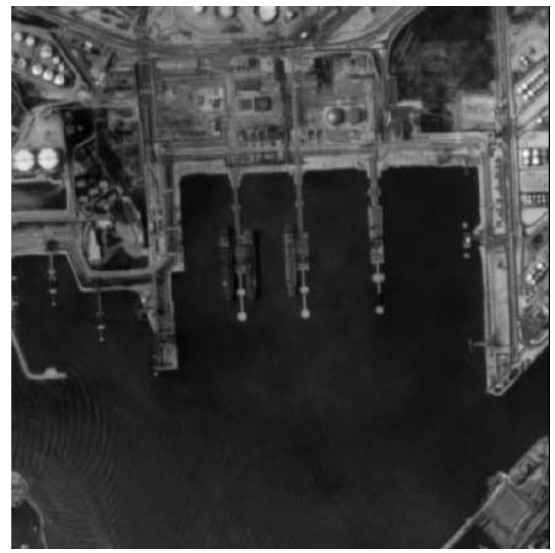

(a)

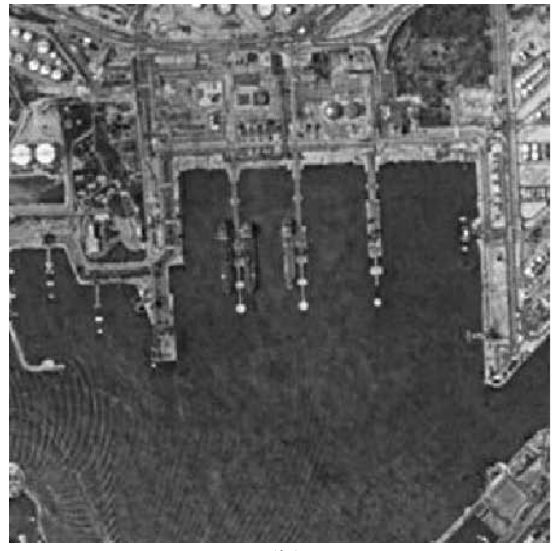

(b)

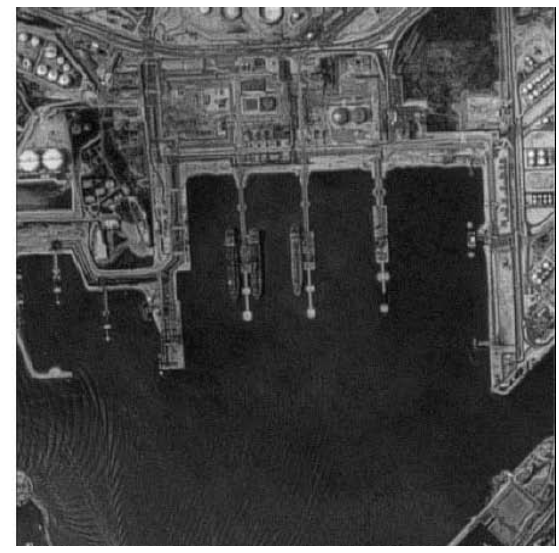

(c)

Fig. 7. (a) Original satellite image. (b) Image enhanced by curvelet method. (c) Image enhanced by block based intensity-pair distribution method. 


\section{Conclusion}

In this paper, we propose a block based intensity-pair distribution method with two expansion forces and linear magnitude mapping function for image contrast enhancement. Since only the block intensity pairs contribute to the intensity mapping function and smooth regions cannot nullify the expansion force of edge pairs, delicate edges are extracted and image enhanced. An additional noise checking procedure has also suppressed noise effectively from input images. Simulation results have shown that the proposed method has done a better improvement compared with the existing methods.

\section{References}

1. Jen, T., Hsieh, B., Wang, S.: Image contrast enhancement based on intensity-pair distribution. In Proc. Int. Conf. Image Processing, Vol. 1, (2005) 913-16

2. Starck, J., Murtagh, F., Candes, E.J., and Donnoho, D.L.: Gray and color image contrast enhancement by the curvelet transform. IEEE Trans. Image Processing, Vol. 12, No. 6, (2003) 706-717

3. Velde, K.V.: Multi-scale color image enhancement. In Proc. Int. Conf. Image Processing, Vol. 3, (1999) 584-587

4. Pei, S.C., Zeng, Y.C., Chang, C.H.: Virtual restoration of ancient Chinese paintings using color contrast enhancement and lacuna texture synthesis. IEEE Trans. Image Processing, Vol. 13, (2004) 416-429

5. Chin, W.A., S.H., Tan, E.C.: Novel approach to automated fingerprint recognition. In Proc. IEE Vision, Image and Signal Processing, Vol. 145, (1998) 160-166

6. Torre, A., Peinado, A.M., Segura, J.C., Perez-Cordoba, J.L., Benitez, M.C., Rubio, A. J.: Histogram equalization of speech representation for robust speech recognition. IEEE Trans. Speech Audio Processing, Vol. 13, (2005) 355-366

7. Pizer, S.M.: The medical image display and analysis group at the university of north carolina: Reminiscences and philosophy. IEEE Trans. Medical Imaging, Vol. 22, (2003) $2-10$

8. Chen, S.-D., Ramli, A.R.: Contrast enhancement using recursive mean-separate histogram equalization for scalable brightness preservation. IEEE Trans. Consumer Electronics, Vol. 49, No. 4, (2003) 1301-1309

9. Gonzalez, R.C., Woods, R.E.: Digital Image Processing. 2nd ed. Reading, MA: AddisonWesley, (1992)

10. Kim, Y.-K.: Contrast enhancement using brightness preserving bi-histogram equalization. IEEE Trans. Consumer Electronics, Vol. 43, No. 1, (1997) 1-8

11. Kim, Y.K., Paik, J.K., Kang, B.S.: Contrast enhancement system using spatially adaptive histogram equalization with temporal filtering. IEEE Trans. on Consumer Electronics, Vol. 44, No. 1, (1998) 82-86

12. Kim, J.-Y., Kim, L.-S, Hwang, S.-H.: An advanced contrast enhancement using partially overlapped sub-block histogram equalization. IEEE Trans. Circuits and Systems for Video Technology, Vol.11, (2001) $475-484$ 